Revista Universo Contábil, ISSN 1809-3337

FURB, v. 5, n.3, p. 116-138, jul./set., 2009

doi:10.4270/ruc.2009325

Disponível em www.furb.br/universocontabil

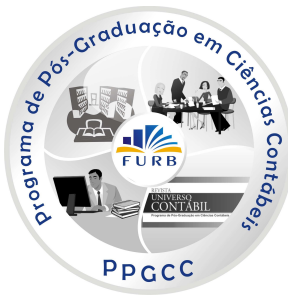

\title{
CAUSAS DE VARIACIÓN DEL RESULTADO CONTABLE ORDINARIO: EL CASO IBERIA'
}

\section{ACCOUNTING OPERATING INCOME CAUSES OF VARIATION: THE IBERIA CASE}

\section{Manuel Angel Fernández Gámez}

Doutor em Ciencias Económicas por la Universidad de Málaga Profesor de la Facultad de Ciencias Económicas y Empresariales da Universidad de Málaga Endereço: Campus El Ejido, s/n. CEP: 29071 - Málaga - España

E-mail: mangel@uma.es

Telefono: (34) 952131234

Daniel Ruiz Palomo

Doutor em Ciencias Económicas por la Universidad de Málaga Profesor de la Facultad de Ciencias Económicas y Empresariales

da Universidad de Málaga Endereço: Campus El Ejido, s/n. CEP: 29071 - Málaga - España

E-mail: drp@uma.es

Telefono: (34) 952137247

\section{Rocío Sánchez Toledano}

Doutoranda em Ciencias Económicas por la Universidad de Málaga Profesora de la Facultad de Ciencias Económicas y Empresariales

da Universidad de Málaga Endereço: Campus El Ejido, s/n. CEP: 29071 - Málaga - España E-mail: mst@uma.es

Telefono.: (34) 952137247

\footnotetext{
Artigo recebido em 14.02.2007. Revisado por pares em 20.11.2007. Reformulado em 30.01.2008. Recomendado em 07.02.2008 por Ilse Maria Beuren (Editora). Publicado em 30.09.2009. Organização responsável pelo periódico: FURB.
} 


\section{RESUMEN}

En el presente artículo pretendemos desarrollar un modelo que permita comprender las causas de variación del resultado contable ordinario y que resulte de aplicación con información externa. Para ello, nos basamos en el estudio de los efectos que producen sobre el mismo una variación de la actividad, del margen de contribución total, de otros ingresos independientes a la actividad, y de los costes fijos. Dada la estrecha relación existente entre actividad y margen de contribución total, analizamos el efecto sobre el resultado ordinario que producen las variaciones de cada uno de sus componentes - costes variables unitarios y tasa de margen -. Con el modelo planteado, de tipo algebraico y discreto, se facilita el estudio del resultado contable ordinario, pues su medición y captación contable se efectúa normalmente por periodos anuales, demasiado amplios para la aplicación de técnicas instrumentales tales como ecuaciones diferenciales o derivadas parciales, de modo que se ha renunciado a la robustez matemática del modelo en aras de su sencillez.

Palabras clave: Resultado operativo. Análisis económico. Variación del resultado. Iberia.

\section{RESUMO}

No presente artigo pretendemos desenvolver um modelo que permita compreender as causas da variação do resultado contábil operacional e que resulte de aplicação com informação externa. Para tanto, nos baseamos no estudo dos efeitos que produzem sobre o mesmo uma variação da atividade, da margem de contribuição total, das outras receitas que independem da atividade e dos custos fixos. Dada a estreita relação existente entre a atividade e a margem de contribuição total, analisamos o efeito sobre o resultado operacional que produzem as variações de cada um de seus componentes - custos variáveis unitários e a taxa de margem -. Com o modelo proposto, de tipo algébrico e discreto, se proporciona o estudo do resultado contábil operacional, pois sua medição e captação contábil se efetuam normalmente por períodos anuais, amplos demais para a aplicação de técnicas instrumentais tais como equações diferenciais o derivadas parciais, de modo que se renunciou a robustez matemática do modelo em lugar da sua simplicidade.

Palavras-chave: Resultado operacional. Análise econômica. Varição do resultado. Ibéria.

\section{ABSTRACT}

In this article it is intended to develop a model which would help understanding the causes of variation of operational earnings as a result of using external information. To do so, we rely on the study of the effects that lead to a variation of the activity, the total contribution margin, and of other incomes that are independent of activity and fixed costs. Given the close relation between activity and total contribution margin, was analyzed the effect on the operational results that generates variations in each of its components - variable costs per unit and margin rate. With the proposed model, algebraic and discrete type, it is possible to study the operational accounting result since its measurement and accountancy collection are normally done in annual periods, too long for the application of instrumental techniques such as differential equations or partial derivatives, in a way that robustness of the mathematical model was renounced the in favor of its simplicity.

Keywords: Operational results. Economy analysis. Results variation. Iberia. 


\section{INTRODUCCIÓN: OBJETIVOS Y APORTACIONES DEL TRABAJO}

La evolución del resultado ordinario es de vital importancia para evitar posibles situaciones de insolvencia, toda vez que de éste se derivan buena parte de los fondos necesarios para la atención de los pagos comprometidos, además de resultar básica para mejorar los niveles de rentabilidad alcanzados, que permitan a la empresa la remuneración de su estructura de capital en condiciones competitivas. En consecuencia, la disposición de un modelo de determinación de las causas económicas que pueden afectar al mismo puede ser de utilidad tanto para el gestor como para el analista externo. Para el primero de ellos, dentro del ámbito de la gestión, en la toma de decisiones oportunas que permitan mejorar el importe del beneficio anual. Para el segundo de ellos, como complemento en labores tanto predictivas como evaluadoras de aquellas magnitudes relacionadas con el resultado, entre ellas, el análisis de rentabilidad y de solvencia. Predictivas, pues puede utilizarse para predecir la futura evolución que podría seguir tal resultado y, a partir de éste, aproximar la cifra de cash-flow ordinario. Evaluadoras, porque permite conocer qué magnitudes económicas han provocado la evolución experimentada por el resultado ordinario, lo que podría aproximar las posibles causas de un déficit de recursos generados ordinarios, que a su vez pudiera provocar situaciones de insolvencia. En definitiva, consideramos que la correcta evolución de la magnitud contable conocida como resultado ordinario se configura como la variable central para asegurar la supervivencia empresarial.

En este sentido, nuestro objetivo fundamental es aportar un modelo algebraico de estudio de la variación del resultado contable ordinario que permita cuantificar, con información externa, el impacto que ejercen sobre el mismo las variaciones experimentadas por las magnitudes que lo definen. A tal efecto, establecemos una función expresiva del efecto que produce sobre el resultado ordinario la variación de cada uno de sus componentes.

Para ello, se ha estimado más oportuna la utilización de un análisis de tipo discreto, pues, aunque el resultado empresarial es de naturaleza continua, su medición y captación contable se efectúa normalmente por periodos anuales, demasiado amplios para la aplicación de técnicas instrumentales tales como ecuaciones diferenciales o derivadas parciales. Así, se ha elegido un modelo aplicable con información discreta, que no permite el estudio de las variaciones marginales experimentadas por la variable dependiente ante cambios infinitesimales en las variables independientes, de modo que se renuncia quizás a dotar al modelo de mayor robustez matemática, pero a cambio se facilita su aplicación directa con información contable. En este sentido, consideramos que el modelo aquí presentado puede servir de base a futuras investigaciones al objeto de su extensión al análisis continuo mediante la aplicación de las citadas técnicas matemáticas.

Asimismo, dada la naturaleza algebraica del modelo presentado, no se considera necesaria su comprobación mediante procesos estadísticos de regresión, ya que, precisamente por poseer tal naturaleza, no cabe posibilidad de error en la medición de las diferentes magnitudes, ni constituye el objeto del presente trabajo su aplicación empírica al contraste de hipótesis. No obstante, creemos que la aplicación de las variables del modelo propuesto a muestras significativas mediante la utilización de técnicas estadísticas y econométricas permitiría contrastar cuál de las causas de variación del resultado tiene una importancia cuantitativa mayor en una determinada población de empresas, o si existen diferencias territoriales o sectoriales en cuanto a la composición del crecimiento del resultado contable ordinario, o si ésta afecta a otras variables dependientes secundarias, tales como, por ejemplo, la rentabilidad de los accionistas, la solvencia empresarial, o el valor de la empresa en el mercado. Se nos ofrece, por tanto, un amplio campo de investigación empírica en este sentido. 
Un análisis de la variación del resultado en función de actividad, precio y costes fijos ha sido desarrollado por García Martín (1984; 1989), y García y Callejón (2001), cuya estructura fundamental se recoge en la ecuación [1].

$$
R_{1}-R_{0}=t_{a} R_{0}+Q_{1}\left[\left(c v u_{1}-c v u_{0}\right) r_{0}+\left(r_{1}-r_{0}\right) c v u_{0}+\left(c v u_{1}-c v u_{0}\right)\left(r_{1}-r_{0}\right)\right]+C F_{0}\left(t_{a}-p\right)
$$

Siendo:

$R_{x}$ : resultado del ejercicio $\mathrm{x}$.

$t_{a}$ : tasa de variación de la actividad, medida en unidades físicas.

$Q_{x}$ : cantidad vendida, medida en unidades físicas, en el ejercicio x.

$c v u_{x}$ : coste variable unitario, por unidad física vendida en el ejercicio x.

$r_{x}$ : tasa promedio que se aplica al coste variable unitario en el ejercicio x para determinar el margen de contribución unitario en dicho ejercicio.

$C F_{0}$ : importe de los costes fijos totales correspondientes al ejercicio de referencia.

$p: \quad$ tasa de variación de los costes fijos

Se ha utilizado el sistema de notación empleado en el trabajo de García y Callejón (2001); aunque no coincide exactamente con el empleado en los trabajos originales de García (1984; 1989). El modelo que presentamos, si bien se basa en dichos trabajos, permite a nuestro juicio mayor profundidad de análisis, derivado de cuatro aportaciones metodológicas: en primer lugar, se explicitan los efectos conjuntos procedentes de variaciones simultáneas entre actividad y cada componente del margen medio; en segundo lugar, se incorpora el efecto producido por el desigual crecimiento de la cifra de negocios y otros ingresos que no se derivan de la actividad; en tercer lugar, se ha adoptado una forma de análisis en cascada para el margen de contribución; por último, se ha abandonado el enfoque monoproducto para abordar el estudio de la variación del resultado ordinario en empresas con producción múltiple mediante la sustitución de la tasa de actividad medida en unidades físicas por la tasa de variación de ventas.

Hemos estructurado el resto del trabajo en tres apartados: uno, para el desarrollo del marco conceptual; otro, en el que a modo ilustrativo hemos incluido una aplicación del modelo a la empresa Iberia para el ejercicio 2004 utilizando exclusivamente información contable externa, y, por último, otro en el que se exponen las conclusiones del trabajo. En los anexos I y II se recogen, asimismo, las notaciones utilizadas y las demostraciones matemáticas que sustentan el modelo.

\section{MARCO CONCEPTUAL: DETERMINACIÓN DE LAS CAUSAS DE VARIACIÓN DEL RESULTADO ORDINARIO}

Es sabido que el resultado ordinario se compone de las siguientes partidas:

+ Importe Neto de la cifra de negocios, o cifra de ventas.

+ Otros ingresos ordinarios -de explotación y financieros-, cuyo importe no se deriva directamente de la actividad principal, o ingresos accesorios.

- Costes de las ventas, cuya cuantía es directamente dependiente de la actividad comercial, esto es, de carácter variable.

- Otros costes de la explotación y gastos financieros, cuya evolución es independiente de la actividad comercial, esto es, de carácter fijo.

Asimismo, se denomina margen comercial o margen de contribución total a la diferencia entre la cifra de ventas y el coste de las mismas. De este modo, el resultado 
ordinario se compone del margen de contribución total, más los ingresos accesorios menos los costes fijos -ecuación [2]-.

$$
R O=V+I A-C V-C F=M C+I A-C F
$$

Donde:

RO: Resultado ordinario.

V: Ventas, o Importe Neto de la Cifra de Negocios.

IA: Ingresos Accesorios (otros ingresos ordinarios no incluidos en la cifra de negocios).

$\mathrm{CV}$ : Coste de las Ventas.

CF: otros costes distintos a los de las ventas (Costes Fijos, semifijos o variables a saltos). En adelante, se considerarán todos como costes fijos.

MC: Margen Comercial, margen de contribución o margen bruto de contribución

Se pueden definir los conceptos anteriores en términos medios por unidad monetaria vendida. Hablamos de beneficio, margen, ingresos, precios y costes medios por unidad monetaria vendida porque rara vez una empresa podrá considerarse monoproducto. Todos estos conceptos se identifican como su correlativo en la cuenta de resultados pero en minúsculas y suprarayado. Así, el Resultado Ordinario medio obtenido a cada unidad

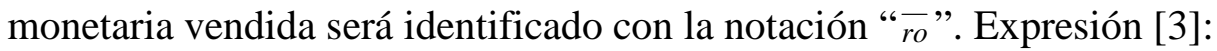

$$
R O=V \overline{r o}=V+V \overline{i a}-V \overline{c v}-V \overline{c f}=V \overline{m c}+V \overline{i a}-V \overline{c f}
$$

Siendo:

RO: Resultado Ordinario

$\mathrm{V}$ : Ventas, o importe neto de la cifra de negocios.

$\overline{r o}$ : cociente entre resultado ordinario y ventas; esto es, importe de resultado ordinario obtenido por término medio a cada unidad monetaria vendida.

$\overline{i a}$ : cociente entre el ingresos accesorios y las ventas; esto es, ingresos accesorios medios respecto a cada unidad monetaria vendida.

$\overline{c v}$ : cociente entre el coste de las ventas y las ventas; esto es, importe del coste de las ventas soportado por cada unidad monetaria vendida.

$\overline{c f}$ : cociente entre los costes fijos y las ventas; esto es, importe del coste fijo recuperado en cada unidad monetaria vendida.

$\overline{m c}$ : cociente entre margen de contribución total y ventas; esto es, importe de margen de contribución obtenido a cada unidad monetaria vendida.

Operando convenientemente se definen las variables que afectan al resultado, relacionando el crecimiento del resultado ordinario con las tasas de variación de las distintas magnitudes que lo componen. Identificamos las tasas de variación con la notación compuesta por la letra " $t$ " seguida de un subíndice expresivo del elemento objeto de cálculo; v.g., la tasa de variación de ventas se denota mediante $t_{V}$, la tasa de variación de los costes fijos totales se denota como $t_{\mathrm{CF}}$, y así sucesivamente. Expresion [4]:

$$
\Delta R O=t_{V} R O_{0}+\left(t_{V}-t_{C F}\right) C F_{0}+\left(t_{I A}-t_{V}\right) I A_{0}+\left(t_{M C}-t_{V}\right) M C_{0}
$$

Donde: 


\begin{abstract}
$\Delta \mathrm{RO}$ : variación del resultado ordinario.
$\mathrm{t}_{\mathrm{V}}$ : tasa de variación de las ventas.

$\mathrm{t}_{\mathrm{IA}}$ : tasa de variación de los ingresos accesorios.

$t_{M C}$ : tasa de variación del margen de contribución total.

$t_{\mathrm{CF}}: \quad$ tasa de variación de los costes fijos.

$\mathrm{RO}_{0}$ : resultado ordinario del ejercicio económico tomado como referencia.

$\mathrm{CF}_{0}$ : costes fijos del ejercicio económico tomado como referencia.

$\mathrm{IA}_{0}$ : ingresos Accesorios del ejercicio económico tomado como referencia.

$\mathrm{MC}_{0}$ : margen de contribución del ejercicio económico tomado como referencia.
\end{abstract}

En consecuencia, puede identificarse qué efecto inflige sobre el resultado ordinario la variación de cada uno de sus componentes -cada uno de los sumandos de la citada expresión-, que analizaremos detenidamente en los apartados siguientes.

\title{
2.1 Variación del Resultado Ordinario por Causa de la Actividad
}

Al objeto de aislar los diferentes efectos provocados por las distintas variables que afectan al resultado ordinario, partimos de unas hipótesis absolutamente restrictivas para relajarlas paulatinamente, identificando así el efecto parcial que causa la relajación de cada una de tales hipótesis al resultado ordinario. Por tanto, ante la inexistencia de costes fijos e ingresos accesorios, o cuando éstos al menos se mantienen constantes por unidad monetaria vendida, y en caso de mantenerse constante la relación existente entre las ventas y todos los componentes del margen de contribución medio por unidad monetaria vendida (coste medio de las ventas y tasa media de margen sobre coste de las ventas), puede definirse la variación del resultado ordinario a consecuencia de un crecimiento o disminución de la actividad como el resultado ordinario del ejercicio anterior multiplicado por la tasa de variación de las ventas.

$$
\begin{array}{ll}
\Delta R O_{V}=t_{V} R O_{0} & \text { Efecto que provoca sobre el resultado ordinario una variación } \\
\text { en la actividad de mantenerse el resultado ordinario medio } \\
\text { constante }
\end{array}
$$

Siendo:

$\Delta R O_{V}$ : variación del resultado ordinario derivado exclusivamente de las ventas, de mantenerse constantes el resto de las magnitudes unitarias.

$t_{V}: \quad$ tasa de variación de las ventas

$R O_{0}$ : resultado ordinario del ejercicio de referencia.

Si todas las partidas que componen el resultado ordinario medio -esto es, los ingresos accesorios medios, el precio medio de venta, el coste variable medio, el margen medio de contribución y el coste fijo medio-, permaneciesen constantes, el resultado ordinario total crecería por importe igual al resultado ordinario medio que aporta cada unidad monetaria vendida más.

A continuación relajaremos paulatinamente las hipótesis planteadas para, uno a uno, aislar los diferentes efectos que provocan las distintas variables consideradas independientes por el modelo sobre el resultado ordinario.

\subsection{Variación del Resultado por Causa de los Costes Fijos}

En primer lugar, relajaremos la hipótesis de inexistencia de costes fijos, o lo que es lo mismo, la hipótesis de que todos los costes crecen de forma proporcional al crecimiento de las 
ventas. Efectivamente, en el corto plazo, los costes fijos no dependen del nivel de actividad. Antes al contrario, su evolución es totalmente independiente de éstos, dependiendo de otros factores, tales como la revisión de las condiciones contractuales que los generan (p.e., la subida de tipos de interés de la deuda), cambios en la capacidad productiva, etc. Por consiguiente, y con independencia de la identificación de dichas causas, si los costes fijos evolucionan a una tasa de crecimiento inferior a la experimentada por las ventas, el resultado ordinario medio obtenido a cada unidad monetaria vendida crecerá en proporción a la diferencia entre tales tasas de crecimiento, y viceversa. Esta relación se expresa a continuación.

$$
\begin{aligned}
\Delta R O_{C F}=\left(t_{V}-t_{C F}\right) C F_{0} & \begin{array}{l}
\text { Efecto que provoca sobre el resultado ordinario una variación } \\
\text { de los costes fijos totales a un ritmo diferente al de la } \\
\text { actividad. }
\end{array}
\end{aligned}
$$

Siendo:

$\Delta R O_{C F}$ : variación del resultado ordinario a consecuencia de los costes fijos.

$t_{V}: \quad$ tasa de variación de las ventas

$t_{C F}: \quad$ tasa de variación de los costes fijos totales

$C F_{0}: \quad$ costes fijos del ejercicio de referencia.

Al ser los costes fijos independientes de la actividad, presentarán una evolución diferente a ésta; de este modo, si su crecimiento es superior al de las ventas, el coste fijo medio que debe cubrir cada unidad monetaria vendida aumentará, lo que hace disminuir el resultado ordinario unitario medio y, por ende, el resultado ordinario total.

Obviamente, al aumentar los costes fijos totales disminuirá el resultado, y viceversa. Sin embargo, en el modelo que se propone los costes fijos tendrán un efecto expansivo sobre el resultado ordinario siempre que su crecimiento sea menor que el de las ventas, dado que se toma como referencia la variación del resultado derivado de la actividad. Lo que se pretende, por tanto, es saber si la existencia de costes fijos favorece o no el crecimiento del resultado respecto al que se obtendría si todos los costes fuesen variables. En este sentido, un crecimiento de los costes fijos a un ritmo inferior al crecimiento de las ventas conlleva que sea necesario aplicar un menor porcentaje de cada unidad monetaria vendida a recuperar el importe de los costes fijos; de este modo, el beneficio obtenido a cada unidad monetaria de ventas aumenta.

Esta propuesta, formalizada por García Martín (1989), muestra el efecto que inflige sobre el resultado un comportamiento de los costes fijos diferente al comportamiento de la actividad. Este autor utiliza como referencia en la medición de la actividad el número de unidades físicas vendidas, determinando la variación experimentada por el resultado que se deriva del comportamiento de los costes fijos para empresas monoproductos. Además, considera que este efecto es explicativo de las causas que justifican el apalancamiento operativo empresarial. De modo que pueden establecerse los siguientes escenarios:

- Si la actividad crece más que los costes fijos totales, el coste fijo medio disminuye, provocando que el resultado medio aumente más de lo que lo haría si todos los costes fuesen variables. Por tanto, el comportamiento de los costes fijos tiene carácter expansivo sobre el resultado ordinario.

$$
t_{V}>t_{C F} \rightarrow\left[\Delta R O_{C F}=\left(t_{V}-t_{C F}\right) C F_{0}\right]>0 \rightarrow\left[\Delta R O=\Delta R O_{V}+\Delta R O_{C F}\right]>\Delta R O_{V}
$$


- En caso contrario, si los costes fijos totales aumentan más que la actividad, el resultado medio se ve mermado, aumentando el resultado total menos de lo que cabría esperar por el aumento de la actividad. Por tanto, el comportamiento de los costes fijos tiene carácter contractivo sobre el resultado ordinario.

$$
t_{V}<t_{C F} \rightarrow\left[\Delta R O_{C F}=\left(t_{V}-t_{C F}\right) C F_{0}\right]<0 \rightarrow\left[\Delta R O=\Delta R O_{V}+\Delta R O_{C F}\right]<\Delta R O_{V}
$$

- Por último, el apalancamiento operativo será neutro cuando los costes fijos totales y la actividad crezcan a idéntico ritmo, pues el resultado medio será constante.

$$
t_{V}=t_{C F} \rightarrow\left(t_{V}-t_{C F}\right) C F_{0}=0 \rightarrow \Delta R O=\Delta R O_{V}
$$

\subsection{Variación del Resultado por Causa de Otros Ingresos Ordinarios Accesorios}

En segundo lugar, relajaremos la hipótesis de inexistencia de ingresos accesorios, o que su crecimiento es proporcional al de las ventas. Al igual que ocurre con los costes fijos, su evolución es absolutamente independiente del nivel de actividad, por lo que consideramos necesario incluir el efecto de tal desviación en el modelo, como se expone a continuación.

$$
\Delta R O_{I A}=\left(t_{I A}-t_{V}\right) I A_{0} \quad \text { Efecto que provoca sobre el resultado ordinario una variación }
$$
de los ingresos accesorios a un ritmo diferente al de la actividad.

Siendo:

$\Delta R O_{I A}$ : variación del resultado ordinario a consecuencia de los ingresos accesorios.

$t_{I A}: \quad$ tasa de variación de los ingresos accesorios.

$t_{V}: \quad$ tasa de variación de las ventas.

$I A_{0}: \quad$ ingresos accesorios del ejercicio de referencia.

Dado que los ingresos accesorios - ingresos financieros y otros ingresos de explotación, fundamentalmente - no emanan de la actividad, mantendrán un crecimiento diferente al de ésta. De esta forma, puede establecerse lo siguiente:

- Si la actividad crece más que los ingresos accesorios totales, el ingreso accesorio medio por unidad monetaria vendida disminuye, por lo que el resultado ordinario medio -y por tanto el total- varían en la misma dirección, ofreciendo esta variación un carácter contractivo.

$t_{V}>t_{I A} \rightarrow\left[\Delta R O_{I A}=\left(t_{I A}-t_{V}\right) I A_{0}\right]<0 \rightarrow\left[\Delta R O=\Delta R O_{V}+\Delta R O_{I A}\right]<\Delta R O_{V}$

- Asimismo, cuando los ingresos accesorios totales aumentan más que la actividad, el resultado medio se ve favorecido por tal crecimiento, aumentando el resultado ordinario en términos absolutos por encima del que se deriva de la actividad. Por tanto, los ingresos accesorios tienen carácter expansivo.

$$
t_{V}<t_{I A} \rightarrow\left[\Delta R O_{I A}=\left(t_{I A}-t_{V}\right) I A_{0}\right]>0 \rightarrow\left[\Delta R O=\Delta R O_{V}+\Delta R O_{I A}\right]>\Delta R O_{V}
$$


- Por último, tendrán carácter neutro cuando actividad e ingresos accesorios crezcan a idéntico ritmo.

$t_{V}=t_{I A} \rightarrow\left(t_{I A}-t_{V}\right) I A_{0}=0 \rightarrow \Delta R O=\Delta R O_{V}$

\subsection{Variación del Resultado por Causa del Margen de Contribución Total}

Si las partidas que componen el margen de contribución total crecen a un ritmo diferente al crecimiento de la actividad, provocarán una modificación en el margen de contribución unitario, lo que a su vez provocará una disminución en el resultado ordinario unitario.

$$
\begin{aligned}
\Delta R O_{M C}=\left(t_{M C}-t_{V}\right) M C_{0} & \begin{array}{l}
\text { Efecto que provoca sobre el resultado ordinario una variación } \\
\text { del margen de contribución total a un ritmo diferente al de la } \\
\text { actividad. }
\end{array}
\end{aligned}
$$

Siendo:

$\Delta R O_{M C}$ : variación del resultado ordinario a consecuencia del margen de contribución total.

$t_{V}: \quad$ tasa de variación de las ventas

$t_{M C}: \quad$ tasa de variación del margen de contribución total

$M C_{0}$ : margen de contribución total del ejercicio de referencia.

Como se ha descrito en la ecuación [4], el resultado depende fundamentalmente de la actividad, pero no exclusivamente de ella. Así, se ha analizado en apartados anteriores qué efecto provoca una variación de los costes fijos totales y/o de los ingresos no incluidos en la cifra de negocios (que hemos denominado ingresos accesorios) diferente al crecimiento de la actividad. En el presente apartado se relaciona el efecto que provoca sobre el resultado ordinario una variación en el margen de contribución medio por unidad monetaria vendida. Para ello, podría realizarse en este apartado un análisis de comportamiento expansivo vs. contractivo similar al expuesto en apartados anteriores, al resultar su expresión isomorfa al resto de sumandos de la expresión [4]. Sin embargo, a nuestro juicio dicho estudio resultaría insuficiente, pues el margen de contribución total depende directamente de la actividad y de la capacidad de gestión de la empresa, por lo que hemos de tratar de discernir qué otras variables inciden sobre él.

En esta línea de argumentación, podemos establecer la variación del resultado ordinario derivada de un cambio en el margen de contribución total en función de la variación en el margen de contribución unitario y la cantidad vendida, de acuerdo a las expresiones [11] y [12], esta última definida en función de tasas de variación.

$$
\begin{aligned}
V R O_{M C} & =\left(\overline{m c_{1}}-\overline{m c_{0}}\right) V_{0}+\left(\overline{m c_{1}}-\overline{m c_{0}}\right)\left(V_{1}-V_{0}\right) \\
\Delta R O_{M C} & =t_{\overline{m c}} M C_{0}+t_{\overline{m c}} t_{V} M C_{0}
\end{aligned}
$$

Por tanto, pueden distinguirse dentro del efecto causado por el margen de contribución total dos efectos diferentes: el provocado por un cambio en el margen de contribución medio, y un efecto conjunto residual, sinérgico o adicional provocado por el cambio simultáneo de la actividad y el margen medio. 
Sean:

$\Delta R O_{\overline{m c}}$ : variación del resultado ordinario a consecuencia del margen de contribución obtenido a cada unidad monetaria vendida.

$\Delta R O_{\overline{m c} ; V}$ : variación del resultado ordinario adicional a consecuencia del crecimiento conjunto de las ventas y el margen de contribución medio por unidad monetaria vendida.

$t_{\overline{m c}}: \quad$ tasa de variación del margen medio de contribución por unidad monetaria vendida

$t_{V}: \quad$ tasa de variación de ventas

$M C_{0}$ : margen de contribución total (en términos absolutos) del ejercicio de referencia.

\subsubsection{Variación del resultado ordinario como consecuencia de un cambio en el margen de contribución medio}

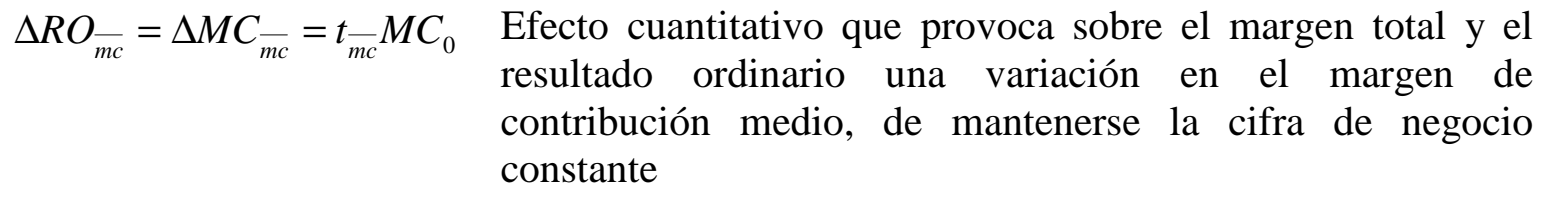

Si se produjese variación en algún sentido en el margen de contribución medio que se obtiene a cada unidad monetaria vendida manteniéndose constante el volumen de ventas, crecería el margen de contribución total, lo que provoca a su vez un crecimiento del resultado ordinario por tal causa.

\subsubsection{Variación del resultado ordinario derivado de un cambio conjunto en el margen de contribución medio y la actividad}

$$
\begin{aligned}
& \Delta R O_{\overline{m c} ; V}=\Delta M C_{\overline{m c} ; V}=t_{\overline{m c}} t_{V} M C_{0} \quad \text { Efecto cuantitativo adicional provocado sobre el } \\
& \text { margen de contribución medio y en la cifra de negocio }
\end{aligned}
$$

Ante variaciones conjuntas de dos elementos, en este caso actividad y margen de contribución medio, se produce un efecto sinérgico que, en el caso de crecimiento de ambas magnitudes, actúa como potenciador del mismo, de modo que el crecimiento del resultado ordinario por tal causa es superior a la suma de ambos efectos por separado.

En la ilustración 1, la superficie del cuadrante inferior izquierdo, delimitada por las coordenadas $\left[\left(0, \mathrm{~V}_{0}\right) ;\left(0, \overline{m c}_{0}\right)\right]$, representa el margen de contribución total del año 0 . Si aumentan las ventas, de $\mathrm{V}_{0}$ a $\mathrm{V}_{1}$, y el margen medio que se obtiene a cada unidad monetaria vendida permanece constante, la variación del resultado vendría delimitada por la superficie de coordenadas $\left[\left(\mathrm{V}_{0}, \mathrm{~V}_{1}\right) ;\left(0, \overline{m c}_{0}\right)\right]$ (cuadrante inferior derecho).

Igualmente, si cambia el margen de contribución medio permaneciendo actividades volumen de ventas constante, la variación del resultado vendría definida por la superficie representada en el cuadrante superior izquierdo, de coordenadas $\left[\left(0, \mathrm{~V}_{0}\right) ;\left(\overline{m c}_{0}, \overline{m c}_{1}\right)\right]$.

Ahora bien, si crecen ambas magnitudes conjuntamente, el margen de contribución total del año 1 vendrá representado por la superficie de coordenadas $\left[\left(0, \mathrm{~V}_{1}\right) ;\left(0, \overline{m c}_{1}\right)\right]$, esto 
es, por los cuatro cuadrantes de la gráfica, siendo su variación la suma de la que proviene de la actividad, más la que proviene del margen de contribución medio, más un efecto conjunto provocado por la variación simultánea entre ambas variables, representado en el cuadrante superior derecho, de coordenadas $\left[\left(\mathrm{V}_{0}, \mathrm{~V}_{1}\right) ;\left(\overline{m c}_{0}, \overline{m c}_{1}\right)\right]$.

En consecuencia, podemos reexpresar la ecuación [4], definitoria de la variación del resultado, como se muestra en la ecuación [13].

$\Delta R O=t_{V} R O_{0}+t_{\overline{m c}} M C_{0}+t_{V} t_{\overline{m c}} M C_{0}+\left(t_{I A}-t_{V}\right) I A_{0}+\left(t_{V}-t_{C F}\right) C F_{0}$

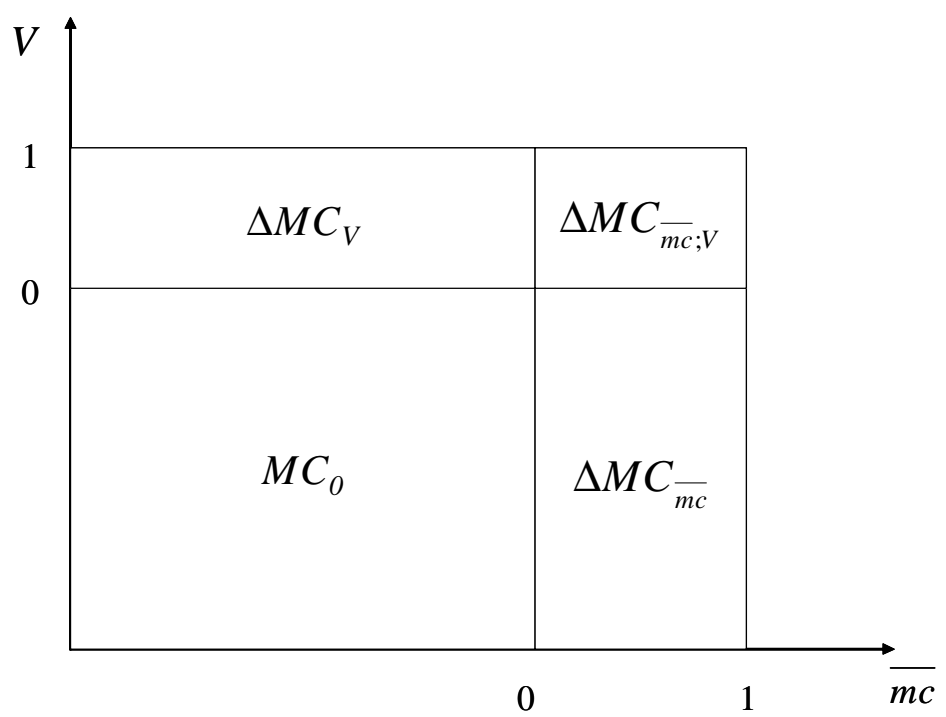

Ilustración 1 - Eefecto conjunto actividad - margen unitario sobre el margen total Fuente: elaboración propia.

\subsection{Identificación de las Causas que Provocan la Variación del Margen Medio de Contribución y su Efecto sobre el Resultado}

El margen de contribución obtenido por término medio a cada unidad física de producto vendida, definido como diferencia entre el precio medio de venta y el coste medio de las ventas, suele fijarse mediante la aplicación de un determinado porcentaje sobre este último, que podemos denominar tasa relativa de margen $(r)$, expresivo del porcentaje de margen obtenido a cada unidad monetaria de coste de las ventas (expresión [14]). Por tanto, tal tasa relativa de margen puede utilizarse para determinar la relación existente entre el margen medio de contribución obtenido y los costes variables medios soportados a unidad monetaria vendida.

$m c u=r \cdot c v u \rightarrow r=\frac{m c u}{c v u}=\frac{M C}{C V}=\frac{\overline{m c}}{\overline{c v}}$

Donde:

mcu: margen medio de contribución obtenido a unidad física vendida.

MC: margen de contribución total obtenido en el ejercicio.

$\overline{m c}$ : margen de contribución obtenido por término medio a cada unidad monetaria vendida.

cvu: coste medio de las ventas de cada unidad física vendida. 

$\overline{c v}$ : coste de las ventas soportado por término medio en cada unidad monetaria vendida.
$r$ : tasa relativa de margen sobre coste variable.

De esta forma, se define la variación del margen de contribución medio en función de cambios en la tasa de margen y el coste variable medio, según se muestra en la ecuación [15], que a su vez provocará un cambio en el resultado ordinario en igual sentido -ecuación [16]-.

$$
\begin{aligned}
& \Delta \overline{m c}=t_{\overline{c v}} \overline{m c_{0}}+t_{r} \overline{m c_{0}}+t_{c v} t_{r} \overline{m c_{0}} \\
& \Delta R O_{\overline{m c}}=\Delta M C_{\overline{m c}}=t_{c v} M C_{0}+t_{r} M C_{0}+t_{\overline{c v}} t_{r} M C_{0}
\end{aligned}
$$

Siendo:

$t_{c v}: \quad$ tasa de variación del coste medio de las ventas.

$t_{r}$ : tasa de variación de la tasa relativa de margen.

$\overline{m c_{0}}$ : margen medio de contribución por unidad monetaria vendida del ejercicio de referencia.

$M C_{0}$ : margen de contribución total del ejercicio de referencia.

Pueden identificarse, consecuentemente, tres causas de variación del margen medio de contribución y del resultado ordinario, manteniendo constantes la actividad y el resto de variables:

$$
\overline{\Delta m c_{c v}}=t_{c v} \overline{m c_{0}}
$$

Efecto que provoca sobre el margen de contribución medio un cambio en el coste medio de las ventas por u.m. vendida, de mantenerse constante la tasa relativa de margen.

De mantenerse constante la tasa de margen, un aumento del coste variable medio hace que, por aumentar su base de cálculo, aumente el margen medio de contribución, lo que a su vez afecta positivamente al resultado ordinario.

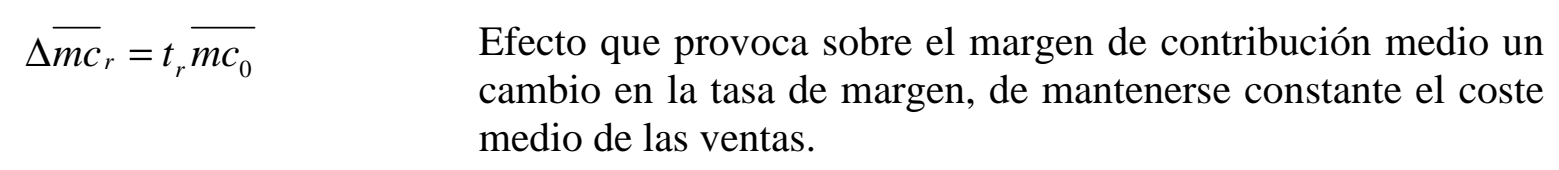

De mantenerse constante el coste variable medio, al aumentar la tasa que se aplica sobre el mismo para determinar el margen medio de contribución, hace que éste aumente, lo que a su vez afecta positivamente al resultado ordinario.

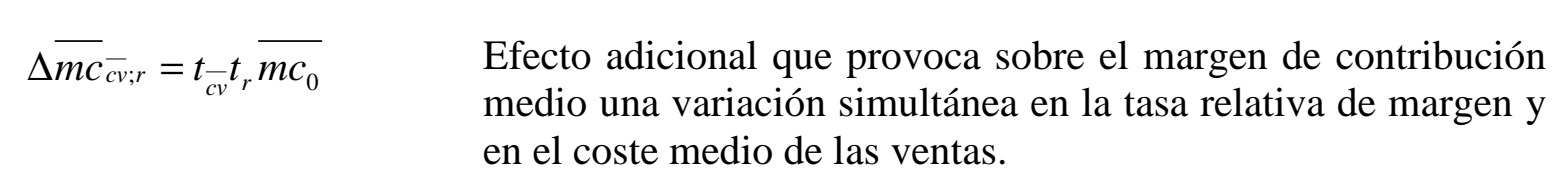

$\mathrm{Al}$ aumentar conjuntamente la tasa relativa de margen " $\mathrm{r}$ ", y la base de cálculo sobre la que se aplica, el margen de contribución medio aumentará en un importe mayor a la suma de los dos efectos tomados independientemente.

De acuerdo con la ecuación [16], si multiplicamos cada uno de estos efectos por las ventas del año 0 , obtendremos la variación del margen de contribución total derivada de los 
cambios en los componentes del margen medio de contribución, de mantenerse la actividad constante, que a su vez representa la variación del resultado ordinario si se mantuviesen constantes el resto de magnitudes medias.

$\Delta R O_{\overline{m c}}=\Delta M C_{\overline{m c}}=t_{c v} M C_{0}+t_{r} M C_{0}+t_{c v} t_{r} M C_{0}$

\subsection{Hacia una Ecuación Global para la Determinación de las Causas de Variación del Resultado Ordinario}

Hasta este momento se han expuesto, para mayor claridad de su sentido económico, análisis parciales del efecto sobre el resultado ordinario provocado por la evolución de las magnitudes que lo conforman, manteniendo en todo el momento la hipótesis de constancia de alguna(s) de las variables explicativas -actividad, costes fijos, etc.-. A continuación relajamos completamente la hipótesis de constancia, obteniendo la ecuación [17], reexpresión de las ecuaciones [4] y [13], para así definir la variación del resultado ordinario como consecuencia de cambios conjuntos en todas las variables analizadas.

$$
\begin{aligned}
& \Delta R O=t_{V} R O_{0}+\left(t_{V}-t_{C F}\right) C F_{0}+\left(t_{I A}-t_{V}\right) I A_{0}+\left\{\left(t_{M C}-t_{V}\right) M C_{0}\right\}= \\
& \left.\Delta R O=t_{V} R O_{0}+\left(t_{V}-t_{C F}\right) C F_{0}+\left(t_{I A}-t_{V}\right) I A_{0}+\left\{t_{\overline{m c}} M C_{0}\right]+\left[t_{V} t_{\overline{m c}} M C_{0}\right]\right\}= \\
& \Delta R O=t_{V} R O_{0}+\left(t_{V}-t_{C F}\right) C F_{0}+\left(t_{I A}-t_{V}\right) I A_{0_{0}}+\left(t_{c v}+t_{r}+t_{c v} t_{r}\right) M C_{0}+\left[t_{V}\left(t_{c v}+t_{r}+t_{c v} t_{r}\right) M C_{0}\right]
\end{aligned}
$$

De estas ecuaciones se desprende que el resultado ordinario puede verse afectado por las siguientes causas:

1. El crecimiento de la actividad $\left(\Delta R O_{V}\right)$

2. Un crecimiento del coste fijo total distinto al crecimiento de la actividad $\left(\triangle R O_{C F}\right)$

3. Un crecimiento de los ingresos accesorios distinto al crecimiento de la actividad $\left(\triangle R O_{I A}\right)$

4. Un crecimiento del margen de contribución total a un ritmo diferente a la actividad $\left(\Delta R O_{M C}\right)$, lo que se puede separar en:

4.1. El crecimiento del margen de contribución medio ( $\Delta R O_{\overline{m c}}$ ), que a su vez incluye:

4.1.1. El crecimiento del coste medio de las ventas $\left(\Delta R O_{\overline{c v}}\right)$

4.1.2. El crecimiento de la tasa relativa de margen $\left(\Delta R O_{r}\right)$

4.1.3. El aumento adicional derivado del crecimiento conjunto de ambas magnitudes $\left(\Delta R O_{\overline{c v} ; r}\right)$.

4.2. El aumento adicional derivado del crecimiento conjunto del margen medio de contribución y la actividad ( $\Delta R O_{\overline{m c} ; V}$ ), que a su vez se desdobla en:

4.2.1. El aumento adicional derivado del crecimiento conjunto de actividad y coste medio de las ventas $\left(\Delta R O_{\overline{c v} ; V}\right)$

4.2.2. El aumento adicional derivado del crecimiento conjunto de actividad y tasa relativa de margen $\left(\Delta R O_{r ; V}\right)$

4.2.3. El aumento adicional derivado del crecimiento conjunto de actividad, coste medio de las ventas y tasa relativa de margen $\left(\Delta R O_{\overline{c v} ; r ; V}\right)$ 


\subsection{Algunas Consideraciones Empíricas para la Aplicación del Modelo}

Indudablemente, el modelo expuesto alcanza todo su sentido cuando se considera la actividad empresarial medida en unidades físicas de producto vendidas en lugar de unidades monetarias vendidas. Sin embargo, este enfoque presenta importantes limitaciones a sus aplicaciones prácticas, que exponemos a continuación, y que nos han hecho inclinarnos a utilizar como indicador de la actividad la variación de las ventas:

a) falta de información externa detallada acerca de las unidades físicas vendidas;

b) falta de homogeneidad de las unidades físicas para la comparabilidad intersectorial;

c) ineficiencia de la utilización de las unidades físicas en el tratamiento masivo de datos;

d) falta de información externa detallada acerca de las partidas que conforman los costes variables unitarios;

e) las empresas raramente son monoproducto.

En primer lugar, el analista se enfrenta al estudio de la cuenta de resultados generalmente desde una perspectiva externa, esto es, con información limitada. En estos casos, no se dispone de información en unidades físicas sobre la producción, que, además, suele ser multiproducto. Para solventar este problema, la doctrina contable propone tres posibilidades: (i) trabajar con cestas de productos; (ii) utilizar un input común a todos los productos; y (iii) medir la actividad a través del volumen de ventas.

Alternativamente, puede tomarse como referencia algún índice de actividad publicado por la propia empresa - por ejemplo, el número de pacientes atendidos para un centro de salud, el número de pernoctaciones para una empresa hotelera, el peso de la basura recogida para una empresa de limpieza, el número de pasajeros para una empresa de transporte, etc. - . Sin embargo, estos índices no siempre están disponibles, y su uso, al igual que las dos primeras soluciones doctrinales recogidas anteriormente, resulta ineficiente para el análisis de muestras significativas cuando se trata de contrastar hipótesis mediante el tratamiento masivo de datos. Estas razones, junto a su disponibilidad y facilidad de medición, nos han inclinado a utilizar la variación de las ventas como indicador de la variación de la actividad.

Igualmente, al no disponerse de información segmentada para cada cesta de productos, no es posible utilizar el análisis propuesto para evaluar la gestión de la cartera de productos. De este modo, el analista externo no podrá aprovechar todo el potencial de análisis que esta herramienta proporciona, debiendo limitarse a valores medios. Será, por consiguiente, en el seno de la contabilidad de gestión donde pueda aplicarse con toda su eficacia este instrumento, desarrollando la formulación apuntada para una cesta de productos y otra de factores variables.

Aranda y Diéguez (2006) han desarrollado un modelo similar al apuntado, para cestas de " $n$ " productos y " $m$ " factores, en el que se discrimina, dentro del efecto actividad, el efecto de la cantidad total vendida más el efecto de un cambio en el mix de productos; asimismo, en el efecto coste variable medio, se distinguen los efectos productividad técnica, productividad económica y cambio en el mix de factores. En este sentido, y obviando estos últimos efectos, si se desea definir la tasa de actividad en función de las unidades físicas vendidas en una empresa multiproducto, pueden redefinirse las siguientes tasas de variación como sigue:

$$
t_{Q}=\frac{\sum_{i=1}^{n} \frac{Q_{1 i}-Q_{0 i}}{Q_{0 i}}}{n} \quad t_{\overline{m c}}=\frac{\sum_{i=1}^{n} \frac{m c u_{1 i}-m c u_{0 i}}{m c u_{0 i}}}{n} \quad t_{\overline{c v}}=\frac{\sum_{i=1}^{n} \sum_{j=1}^{m} \frac{c v_{1 i j}-c v_{0 i j}}{c v_{0 i j}}}{n}
$$


Por otra parte, el problema de más difícil solución es estimar, a partir de la información externa y resumida que se presenta en la cuenta de resultados, qué gastos son reflejo de costes fijos y cuáles de costes variables. Al no disponerse de información suficiente, no se puede distinguir correctamente entre unos y otros. No obstante, desde un punto de vista conceptual, el análisis apuntado adquiere sentido si consideramos como coste variable exclusivamente al coste de las ventas (GARCÍA; CALLEJÓN, 2001), pues es la única rúbrica contable externa en la que puede establecerse con seguridad un criterio racional de causaefecto respecto a la actividad.

Por último, queremos apuntar que el analista externo puede determinar la tasa relativa de margen " $r$ " a partir del margen de contribución total y el coste variable total, pues el efecto actividad queda anulado por encontrarse simultáneamente en el numerador y el denominador.

\section{UN ESTUDIO DEL CASO: IBERIA}

\subsection{Hipótesis, Alcance y Limitaciones del Trabajo}

Para determinar el nivel de actividad, se ha utilizado como indicador la cifra de negocio de Iberia durante dos años consecutivos. En consecuencia, al carecer de información, no hemos hecho distinción entre las líneas de vuelo existentes. Al no poder distinguir la aportación de las líneas mantenidas por la empresa, no podemos concluir cuál o cuáles de ellas afectan positiva o negativamente al resultado. Asimismo, los resultados medios obtenidos son promedios simples de cada una de las variables analizadas. En la Tabla 1 se muestra el volumen total de ventas de los ejercicios 2003 y 2004 , medidos en miles de euros.

Tabla 1 - Indicador de actividad utilizado y su evolución (miles de euros)

\begin{tabular}{l|c|c|c|c}
\hline & $\mathbf{2 0 0 4}$ & $\mathbf{2 0 0 3}$ & Variación & Tasa var. \\
\hline Importe neto de la cifra de negocios & 4.523 .894 & 4.321 .560 & 202.334 & $4,68 \%$ \\
\hline
\end{tabular}

Fuente: cuentas anuales de Iberia.

Como se ha explicado, el análisis efectuado alcanza exclusivamente al resultado contable ordinario, quedando excluido del mismo, entre otras partidas, el impuesto sobre el beneficio. No obstante, somos conscientes que determinados gastos ajenos al resultado ordinario podrían formar parte de los costes estructurales de la empresa y que, en consecuencia, son incorporados al coste del producto. En la Tabla 2 se muestra el resultado ordinario de los ejercicios 2003 y 2004. Dentro de éste, se han tomado como referencia de los ingresos derivados de la actividad el importe neto de la cifra de negocios, y como ingresos ordinarios accesorios la agregación de ingresos financieros y otros ingresos de explotación.

Tabla 2 - Resultado ordinario de Iberia (miles de euros)

\begin{tabular}{l|r|r|l|r|r}
\hline GASTOS & $\mathbf{2 0 0 4}$ & $\mathbf{2 0 0 3}$ & INGRESOS & $\mathbf{2 0 0 4}$ & $\mathbf{2 0 0 3}$ \\
\hline Aprovisionamientos & 849.592 & 737.012 & I. Neto Cifra Negocio & 4.523 .894 & 4.321 .560 \\
Gastos de personal & 1.411 .807 & 1.355 .716 & Otros ingresos explot. & 215.723 & 229.208 \\
Dot. Amortizaciones & 183.158 & 173.382 & & & \\
Var. provis. Tráfico & 4.874 & 592 & & & \\
otros gastos explot. & 2.103 .594 & 2.144 .905 & & & \\
\hline Gastos explotación & 4.553 .025 & 4.411 .607 & Ingresos explotación & 4.739 .617 & 4.550 .768 \\
\hline Beneficio explotación & $\mathbf{1 8 6 . 5 9 2}$ & $\mathbf{1 3 9 . 1 6 1}$ & & & \\
Gastos Financieros & 123.538 & 156.875 & Ingresos Financieros & 154.702 & 164.027 \\
\hline Beneficio financiero & $\mathbf{3 1 . 1 6 4}$ & $\mathbf{7 . 1 5 2}$ & & & \\
\cline { 1 - 3 } Resultado Ordinario & $\mathbf{2 1 7 . 7 5 6}$ & $\mathbf{1 4 6 . 3 1 3}$ & & & \\
\cline { 1 - 3 }
\end{tabular}

Fuente: cuentas anuales de Iberia. 
Respecto a la discriminación de cada uno de los componentes de los gastos en fijos o variables, hemos considerado variables los costes por aprovisionamientos $\mathrm{y}$, según se desprende de la información contenida en la memoria, parte de los gastos contenidos en la rúbrica Otros Gastos de Explotación. En la Tabla 3 se muestra la discriminación efectuada en ella. Así, el coste fijo se ha determinado por agregación del total de gastos de explotación restante -gastos de personal, dotación de amortizaciones, variación de provisiones del tráfico y la parte restante de otros gastos de explotación- más los gastos financieros.

Tabla 3 - Composición de la partida Otros Gastos de Explotación (miles de euros)

\begin{tabular}{l|r|r}
\hline \multicolumn{1}{c|}{ OTROS GASTOS DE EXPLOTACIÓN } & $\mathbf{2 0 0 4}$ & $\mathbf{2 0 0 3}$ \\
\hline Gastos comerciales & 1.212 .560 & 1.290 .937 \\
Servicios tráfico aéreo & 342.406 & 437.828 \\
Gastos de escala & 359.145 & 332.247 \\
Tasas de navegación & 41.162 & 40.850 \\
Gastos de sistema de reservas & 270.533 & 257.593 \\
Servicios a bordo & 130.347 & 128.164 \\
& 68.967 & 94.255 \\
\hline Alquiler flota aérea (leasing) & 891.034 & 853.968 \\
cobertura tipos interés alquiler & 319.926 & 325.485 \\
Incidencias & 43.616 & 22.758 \\
Mantenimiento flota & 16.895 & 12.867 \\
Otro mantenimiento & 117.163 & 104.708 \\
Otros alquileres y otros & 33.667 & 30.344 \\
\hline TOTAL & 359.767 & 357.806 \\
\hline Futen & 2.103 .594 & 2.144 .905 \\
\hline
\end{tabular}

Fuente: elaboración propia a partir de las cuentas anuales de Iberia.

Debido a las limitaciones anteriores, somos conscientes de que el alcance del presente trabajo es ofrecer una visión de la aplicabilidad práctica del modelo que proponemos, sin estar en nuestro ánimo asegurar la completa fiabilidad de las conclusiones obtenidas del estudio empírico.

\subsection{Aplicación del Modelo y Resultados Obtenidos}

La Tabla 4 muestra la adecuación de la cuenta de resultados ordinarios para nuestro análisis.

Tabla 4 - Adecuación del resultado ordinario de Iberia en 2004 y 2003 (miles de $€$ )

\begin{tabular}{l|r|r|l|r|r}
\hline GASTOS & $\mathbf{2 0 0 4}$ & $\mathbf{2 0 0 3}$ & INGRESOS & $\mathbf{2 0 0 4}$ & $\mathbf{2 0 0 3}$ \\
\hline G. VARIABLES & $\mathbf{2 . 0 6 2 . 1 5 2}$ & $\mathbf{2 . 0 2 7 . 9 4 9}$ & VENTAS & $\mathbf{4 . 5 2 3 . 8 9 4}$ & $\mathbf{4 . 3 2 1 . 5 6 0}$ \\
Aprovisionamientos & 849.592 & 737.012 & Cifra de negocios & 4.523 .894 & 4.321 .560 \\
otros G. expl., vbles. & 1.212 .560 & 1.290 .937 & & & \\
\hline G. FIJOS & $\mathbf{2 . 6 1 4 . 4 1 1}$ & $\mathbf{2 . 5 4 0 . 5 3 3}$ & I. ACCESORIOS & $\mathbf{3 7 0 . 4 2 5}$ & $\mathbf{3 9 3 . 2 3 5}$ \\
Gastos de personal & 1.411 .807 & 1.355 .716 & Otros I. explot. & 215.723 & 229.208 \\
Amortizaciones & 183.158 & 173.382 & I. financieros & 154.702 & 164.027 \\
Var. provis. Tráfico & 4.874 & 592 & & & \\
otros G. expl.,fijos & 891.034 & 853.968 & & & \\
Gastos Financieros & 123.538 & 156.875 & & & \\
\hline Resultado ordinario & $\mathbf{2 1 7 . 7 5 6}$ & $\mathbf{1 4 6 . 3 1 3}$ & & & \\
\hline
\end{tabular}

Fuente: elaboración propia a partir de las cuentas anuales.

En la Tabla 5 se han determinado el importe de las partidas que componen el resultado ordinario de Iberia en 2004 y 2003, y sus tasas de variación. 
Tabla 5 - Magnitudes absolutas definitorias del resultado ordinario total

\begin{tabular}{c|l|r|r|r|r|r}
\hline Notación & \multicolumn{1}{|c|}{ Magnitudes absolutas } & \multicolumn{1}{c|}{$\mathbf{2 0 0 4}$} & \multicolumn{1}{c|}{$\mathbf{2 0 0 3}$} & \multicolumn{1}{c|}{ Var. } & Tasa var. & Notación \\
\hline V & Ventas & 4.523 .894 & 4.321 .560 & 202.334 & $4,68 \%$ & $\mathrm{t}_{\mathrm{V}}$ \\
$\mathrm{CV}$ & Costes de las ventas & 2.062 .152 & 2.027 .949 & 34.203 & $1,69 \%$ & $\mathrm{t}_{\mathrm{CV}}$ \\
$\mathrm{MC}$ & Margen de contribución total & 2.461 .742 & 2.293 .611 & 168.131 & $7,33 \%$ & $\mathrm{t}_{\mathrm{MC}}$ \\
$\mathrm{IA}$ & Ingresos Accesorios & 370.425 & 393.235 & -22.810 & $-5,80 \%$ & $\mathrm{t}_{\mathrm{IA}}$ \\
$\mathrm{CF}$ & Costes fijos & 2.614 .411 & 2.540 .533 & 73.878 & $2,91 \%$ & $\mathrm{t}_{\mathrm{CF}}$ \\
$\mathrm{RO}$ & Resultado Ordinario & 217.756 & 146.313 & 71.443 & $48,83 \%$ & $\mathrm{t}_{\mathrm{RO}}$ \\
\hline
\end{tabular}

Miles de euros, excepto tasas de variación, expresadas en porcentajes.

Fuente: elaboración propia a partir de las cuentas anuales.

En la Tabla 6, las magnitudes unitarias medias por unidad monetaria vendida, para los años 2004 y 2003, y sus tasas de variación correspondientes.

Tabla 6 - Magnitudes medias por unidad monetaria vendida definitorias del resultado ordinario

\begin{tabular}{c|l|r|r|r|r|r}
\hline Notación & \multicolumn{1}{|c|}{ Magnitudes medias } & $\mathbf{2 0 0 4}$ & $\mathbf{2 0 0 3}$ & Variación & Tasa var. & Notación \\
\hline $\mathrm{cv}$ & coste medio de ventas & 0,4558 & 0,4693 & $-0,0134$ & $-2,86 \%$ & $\mathrm{t}_{\mathrm{cv}}$ \\
$\mathrm{mc}$ & margen medio de contribución & 0,5442 & 0,5307 & 0,0134 & $2,53 \%$ & $\mathrm{t}_{\mathrm{mc}}$ \\
$\mathrm{ia}$ & Ingresos accesorios medios & 0,0819 & 0,0910 & $-0,0091$ & $-10,01 \%$ & $\mathrm{t}_{\mathrm{ia}}$ \\
$\mathrm{cf}$ & Costes fijos medios & 0,5779 & 0,5879 & $-0,0100$ & $-1,69 \%$ & $\mathrm{t}_{\mathrm{cf}}$ \\
$\mathrm{ro}$ & resultado ordinario medio & 0,0481 & 0,0339 & 0,0143 & $42,17 \%$ & $\mathrm{t}_{\mathrm{ro}}$ \\
$\mathrm{r}$ & tasa de margen "r" & 1,1938 & 1,1310 & 0,0628 & $5,55 \%$ & $\mathrm{t}_{\mathrm{r}}$ \\
\hline
\end{tabular}

Magnitudes expresadas en tanto por uno respecto a las ventas, excepto tasas de variación

Fuente: elaboración propia a partir de las cuentas anuales.

Una vez obtenidos estos, la obtención desagregada de la variación del resultado expresiva de sus distintas causas se consigue por aplicación directa de la formulación propuesta. En la Tabla 7 hemos recogido el impacto que experimenta el resultado a consecuencia de las variaciones de sus distintos componentes.

Tabla 7 - Impacto sobre el resultado ordinario de la variación de sus componentes

\begin{tabular}{l|c|c|c}
\multicolumn{1}{c|}{ Notación } & Concepto & Miles de euros & Fórmula \\
\hline$\Delta \mathrm{RO}$ & Var. Resultado Ordinario & 71.443 & $\mathrm{RO}_{1}-\mathrm{RO}_{0}$ \\
\hline$\Delta \mathrm{RO}_{\mathrm{V}}$ & - Efecto actividad & 6.850 & $\mathrm{RO}_{0} \mathrm{t}_{\mathrm{V}}$ \\
$\Delta \mathrm{RO}_{\mathrm{IA}}$ & - Efecto Ingresos accesorios & -41.221 & $\mathrm{IA}_{0}\left(\mathrm{t}_{\mathrm{IA}}-\mathrm{t}_{\mathrm{V}}\right)$ \\
$\Delta \mathrm{RO}_{\mathrm{CF}}$ & - Efecto Costes Fijos & 45.069 & $\mathrm{CF}_{0}\left(\mathrm{t}_{\mathrm{V}}-\mathrm{t}_{\mathrm{CF}}\right)$ \\
$\Delta \mathrm{RO}_{\mathrm{MC}}$ & - Efecto Margen Contribución Total & 60.745 & $\mathrm{MC}_{0}\left(\mathrm{t}_{\mathrm{MC}}-\mathrm{t}_{\mathrm{V}}\right)$ \\
$\Delta \mathrm{RO}_{\mathrm{mc}}$ & - Efecto margen medio contrib. & 58.028 & $\mathrm{MC}_{0} \mathrm{t}_{\mathrm{mc}}$ \\
$\Delta \mathrm{RO}_{\mathrm{cv}}$ & - Efecto coste vble medio & -65.630 & $\mathrm{MC}_{0} \mathrm{t}_{\mathrm{cv}}$ \\
$\Delta \mathrm{RO}_{\mathrm{r}}$ & - Efecto tasa de margen & 127.300 & $\mathrm{MC}_{0} \mathrm{t}_{\mathrm{r}}$ \\
$\Delta \mathrm{RO}_{\mathrm{cv} ; \mathrm{r}}$ & - Efecto conjunto cv-r & -3.643 & $\mathrm{MC}_{0} \mathrm{t}_{\mathrm{cv}} \mathrm{t}_{\mathrm{r}}$ \\
$\Delta \mathrm{RO}_{\mathrm{V} ; \mathrm{mc}}$ & - Efecto conjunto mg medio-act & 2.717 & $\mathrm{MC}_{0} \mathrm{t}_{\mathrm{v}} \mathrm{t}_{\mathrm{mc}}$ \\
$\Delta \mathrm{RO}_{\mathrm{V} ; \mathrm{cv}}$ & - Efecto conjunto cv-act & -3.073 & $\mathrm{MC}_{0} \mathrm{t}_{\mathrm{v}} \mathrm{t}_{\mathrm{cv}}$ \\
$\Delta \mathrm{RO}_{\mathrm{V} ; \mathrm{r}}$ & - Efecto conjunto r-act & 5.960 & $\mathrm{MC}_{0} \mathrm{t}_{\mathrm{v}} \mathrm{t}_{\mathrm{r}}$ \\
$\Delta \mathrm{RO}_{\mathrm{V} ; \mathrm{cv} ; \mathrm{r}}$ & - Efecto conjunto cv-r-act & -171 & $\mathrm{MC}_{0} \mathrm{t}_{\mathrm{v}} \mathrm{t}_{\mathrm{cv}} \mathrm{t}_{\mathrm{r}}$ \\
\hline
\end{tabular}

Fuente: elaboración propia.

En dicha tabla puede observarse que el aumento del beneficio ordinario de 7,14 millones de euros se deriva fundamentalmente de la mejora experimentada por la tasa relativa de margen "r", como analizaremos a continuación:

el crecimiento de la actividad, por sí mismo, sólo provocaría un aumento del beneficio en torno a 6,8 millones de euros. 
a) el menor crecimiento de los costes fijos totales respecto a la actividad provoca un efecto expansivo sobre el resultado en torno a 45 millones de euros;

b) el comportamiento contractivo de los ingresos accesorios provoca una disminución del beneficio de 41,2 millones de euros, que prácticamente compensa el carácter expansivo de los costes fijos;

c) al crecer el margen de contribución total más que la actividad, se obtiene un crecimiento del beneficio de 60,7 millones de euros, que a su vez proviene, por importe de 58 millones, del crecimiento de los márgenes medios, y por 2,7 millones del efecto conjunto entre éstos y la actividad;

d) a su vez, este aumento del resultado derivado del margen medio de contribución proviene de un aumento de las tasas de margen que, de haberse mantenido los costes variables medios constantes, hubiese ascendido a 127,3 millones de euros. Sin embargo, el aumento de la tasa de margen está íntimamente relacionado con el descenso de los costes variables medios, toda vez que es este comportamiento el que permite el aumento de aquella.

Por tanto, puede decirse que la empresa ha mejorado el rendimiento obtenido a sus costes variables, y ha podido trasladar a los precios dicha mejora. De no haberse producido dicho traslado, el descenso de los costes variables hubiese provocado un descenso del resultado ordinario de 65,6 millones de euros.

\section{CONCLUSIONES}

Con el modelo planteado, de tipo algebraico y discreto, se facilita el estudio del resultado contable ordinario, pues su medición y captación contable se efectúa normalmente por periodos anuales, demasiado amplios para la aplicación de técnicas instrumentales tales como ecuaciones diferenciales o derivadas parciales, de modo que se ha renunciado a la robustez matemática del modelo en aras de su sencillez. Asimismo, al ser algebraico, no necesita ser contrastado mediante procesos estadísticos de regresión

Respecto a su utilidad, el modelo que proponemos para el análisis de dicho resultado permite al analista externo cuantificar el impacto que ejercen sobre el mismo las variaciones experimentadas por todas las magnitudes que inciden sobre él. En este contexto, nuestra aportación respecto a los modelos pioneros de García Martín $(1984,1989)$ y García y Callejón (2001) ha consistido en:

a) abandonar el enfoque monoproducto, resolviendo el problema mediante la utilización de la tasa de variación de ventas como indicador del crecimiento de la actividad, lo que nos ha permitido a su vez determinar, por unidad monetaria vendida, magnitudes económicas medias y tasas de variación, que facilitan la aplicación del modelo con información externa;

b) cuantificar los efectos conjuntos procedentes de variaciones simultáneas entre la actividad y cada uno de los componentes del margen de contribución medio;

c) incorporar el efecto que inflige el desigual crecimiento de los ingresos derivados de la actividad y aquellos otros que, aún siendo ordinarios, no se derivan de ésta;

d) adoptar un análisis en cascada para el margen de contribución, discriminando:

- el efecto producido sobre el resultado ordinario un crecimiento del margen de contribución diferente al crecimiento de las ventas;

- el efecto del crecimiento del margen de contribución medio por unidad monetaria vendida;

- el efecto conjunto del crecimiento de las ventas y del crecimiento del margen de contribución medio por unidad monetaria vendida

Respecto a otros modelos utilizados convencionalmente por la doctrina, consideramos que nuestra aportación consiste, fundamentalmente, en el desarrollo de un modelo que facilita 
el conocimiento de las causas económicas que provocan una variación del resultado, y que resulta aplicable con información externa. La aplicación de los modelos convencionales de análisis del resultado nos parece insuficiente para explicar en toda su dimensión sus causas de variación, debido sobre todo al uso de hipótesis demasiado restrictivas, o a la necesidad de utilizar información generalmente no disponible para el analista externo.

Respecto a futuros desarrollos del presente trabajo, consideramos que éstos pueden venir por dos vías: una, de investigación básica, que a trate de asentar los fundamentos matemáticos del modelo para su aplicación a variables continuas mediante la utilización de ecuaciones diferenciales; otra, de investigación aplicada, consistente en la aplicación del modelo propuesto al contraste de hipótesis mediante la utilización de técnicas estadísticas y econométricas, entre las que destacan:

a) importancia cuantitativa de los diferentes efectos en la empresa para uno o varios periodos;

b) diferencias significativas de su composición en función de la actividad, el área geográfica o la dimensión;

c) diferencias significativas de su composición en función de la situación de la empresa desde las ópticas económica (rentabilidad), financiera (solvencia, liquidez,...), patrimonial (composición del balance), jurídica (sociedades con responsabilidad limitada vs. ilimitada de sus socios; empresa familiar vs. no familiar; etc.), etc;

d) análisis de los factores determinantes de las principales causas de variación del resultado, etc.

Por último, puede resultar interesante el estudio de los efectos que se provocan sobre el grado de apalancamiento operativo por las diferentes causas de variación del resultado ordinario, y/o el efecto de los diferentes tipos de productividad sobre los diferentes sumandos del modelo, en especial los relativos al margen de contribución y los costes fijos.

\section{REFERENCIAS}

AECA Costes estándares y análisis de desviaciones. Documento $\mathbf{n}^{\mathbf{0}} \mathbf{1 2}$, serie Principios de Contabilidad de Gestión, 1998.

AMERICAN PRODUCTIVITY CENTER. Total performance measurement. Houston: APC, 1981.

ARANDA LLAMAS, E.; DIÉGUEZ SOTO, J. Un análisis del apalancamiento operativo y su relación con el resultado empresarial. Revista Iberoamericana de Contabilidad de Gestión, v. 4, n. 7, ene./jun., 2006.

BANKER, R. D.; DATAR S. M. Accounting for labour productivity in manufacturing operations: an application. Accounting and Management - Field Study Perspectives, edited by W. Bruns and R. S. Kaplan. Boston, MA: Harvard Business School Press: 1987. pp. 169203.

BANKER, R. D.; DATAR S. M.; KAPLAN, R.S. Productivity measurement and management accounting. Journal of Accounting, Auditing \& Finance, p. 528-554, 1989.

BUENAVENTURA, V. El estudio de apalancamientos como metodología de análisis de la gestión de la empresa. Estudios Gerenciales, n. 82, p. 65-91, ene./mar, 2002.

DIEGUEZ SOTO, J. El efecto productividad: un instrumento para el análisis contable. Partida Doble, n. 88, p. 42-65, 1998.

DRURY, C. Standard costing. New York: Academic Press, 1992. 
ESTEO SÁNCHEZ, F. Análisis contable de la rentabilidad empresarial. Madrid: Centro de Estudios Financieros, 1998.

FERNÁNDEZ PIRLA, J.M. Teoría económica de la contabilidad. Madrid: Ed. ICE, 1972.

GARCÍA MARTÍN, V. Causas fundamentales que justifican la variación del resultado de un periodo respecto a otro anterior: su formulación analítica. Revista Técnica Contable, Jul., 1984.

GARCÍA MARTÍN, V. Limitaciones del apalancamiento operativo: una propuesta alternativa. Revista Técnica Contable, ago./sep., 1989.

GARCÍA MARTÍN, V.; CALLEJÓN GIL, A.M. Variación del resultado y apalancamiento operativo. Revista Técnica Contable, diciembre, 2001.

IBERIA. Cuentas anuales. Comisión Nacional del Mercado de Valores, 2003, 2004.

IBERIA. Informe de actividad. Grupo Iberia, 2003, 2004.

KAPLAN, R.S.; ATKINSON, A. Advanced Management Accounting. New York: Prentice Hall, 1989.

MALLADO RODRÍGUEZ, J.A. El apalancamiento operativo como herramienta de gestión. Actualidad Financiera, monográfico, $4^{\circ}$ trimestre 1997.

MORA ENGUÍDANOS, A. El apalancamiento operativo de la empresa: aspectos prácticos y utilidad para la toma de decisiones. Tecnica Contable, v. 47, 1995.

PELES, Y.C. A note on yield variante and mix variante. The Accounting Review, v. 61, n. 2, April, 1986.

PIÑEIRO FERNÁNDEZ, S. Aspectos económico-financieros y el potencial de crecimiento de las empresas en época de crisis. Cuadernos de Estudios Empresariales, n. 6, p. 311-326, 1996.

PRIOR, D. Descentralización y desviaciones en resultados. Congreso AECA, 10., Zaragoza, 1999. Anais ... Zaragoza: AECA, 1999.

RODRÍGUEZ-VILARIÑO, A. Apalancamiento operativo: concepto y clases. Revista Española de Contabilidad y Tributación: comentarios y casos prácticos, n. 159, p. 165192, 1996.

RUEDA, J.A. Respuesta del precio de las acciones a la información aportada por los niveles y componentes del resultado contable. Workshop de Investigación Empírica en Contabilidad Financiera, III, Alicante, 2003. Anais ... Alicante, 2003.

SANCHEZ GARCÍA et al. Método para conocer el grado de apalancamiento financiero y operativo. Estrategia financiera, n. 195, p. 22-29, 2003.

VAZQUEZ ABAD, J. La relación entre la estructura de explotación y la estrategia competitiva de la empresa a través del apalancamiento operativo. Alta Dirección, n. 38, p. 29-36, sep./oct. 2002.

VERGÉS, J. La planificació económica i financiera de la empresa: elaboració y utilització de previsions com a base de la gestió. Barcelona: Universitat Autónoma de Barcelona, servei de publicacions, 1994. 
ANEXO I - NOTACIONES UTILIZADAS

\begin{tabular}{|c|c|c|c|}
\hline Concepto & $\begin{array}{l}\text { magnitud } \\
\text { absoluta }\end{array}$ & $\begin{array}{l}\text { por unidad } \\
\text { monetaria } \\
\text { vendida }\end{array}$ & $\begin{array}{c}\text { Tasas } \\
\text { de variación } \\
\text { utilizadas } \\
\end{array}$ \\
\hline Resultado Ordinario & $R O$ & $\overline{r o}$ & $t_{R O}=\frac{R O_{1}-R O_{0}}{R O_{0}}$ \\
\hline Ventas (cifra de negocios) & $V$ & 1 & $t_{V}=\frac{V_{1}-V_{0}}{V_{0}}$ \\
\hline $\begin{array}{c}\text { Ingresos Accesorios } \\
\text { (ordinarios distintos de las ventas) }\end{array}$ & $I A$ & $i a$ & $t_{I A}=\frac{I A_{1}-I A_{0}}{I A_{0}}$ \\
\hline Coste de las ventas & $C V$ & $\overline{c v}$ & $t_{\overline{c v}}=\frac{\overline{c v}_{1}-\overline{c v}_{0}}{\overline{c v}_{0}}$ \\
\hline Costes fijos & $C F$ & $\overline{c f}$ & $t_{C F}=\frac{C F_{1}-C F_{0}}{C F_{0}}$ \\
\hline $\begin{array}{l}\text { Margen de Contribución o } \\
\text { Margen Comercial }\end{array}$ & $M C$ & $\overline{m c}$ & $\begin{aligned} t_{M C} & =\frac{M C_{1}-M C_{0}}{M C_{0}} \\
t_{\overline{m c}} & =\frac{\overline{m c}_{1}-\overline{m c}_{0}}{\overline{m c}_{0}}\end{aligned}$ \\
\hline Tasa relativa de margen & $r=\frac{M C}{C V}$ & $r=\frac{\overline{m c}}{\overline{c v}}$ & $t_{r}=\frac{r_{1}-r_{0}}{r_{0}}$ \\
\hline
\end{tabular}




\section{ANEXO II - DEMOSTRACIONES MATEMÁTICAS}

Sea el resultado ordinario de dos periodos consecutivos, 0 y 1, y su variación:

$$
\begin{aligned}
& R O_{1}=V_{1}+I A_{1}-C V_{1}-C F_{1}=M C_{1}+I A_{1}-C F_{1} \\
& R O_{0}=V_{0}+I A_{0}-C V_{0}-C F_{0}=M C_{0}+I A_{0}-C F_{0}
\end{aligned}
$$

Tomando diferencias entre ambas y reexpresandolas como tasas de variación multiplicando y dividiendo cada sumando por su componente inicial:

$$
\begin{aligned}
& \Delta R O=R O_{1}-R O_{0}=\left(M C_{1}-M C_{0}\right)+\left(I A_{1}-I A_{0}\right)-\left(C F_{0}-C F_{0}\right)= \\
& \Delta R O=\frac{\left(M C_{1}-M C_{0}\right)}{M C_{0}} M C_{0}+\frac{\left(I A_{1}-I A_{0}\right)}{I A_{0}} I A_{0}-\frac{\left(C F_{0}-C F_{0}\right)}{C F_{0}} C F_{0}= \\
& \Delta R O=t_{M C} M C_{0}+t_{I A} I A_{0}+t_{C F} C F_{0}
\end{aligned}
$$

Como $M C_{0}=R O_{0}-I A_{0}+C F_{0}$, sumando y restando $t_{V} M C_{0}$ a la expresión anterior, y sacando factores comunes:

$$
\begin{aligned}
& \Delta R O=t_{M C} M C_{0}+t_{V} M C_{0}-t_{V} M C_{0}+t_{I A} I A_{0}-t_{C F} C F_{0}=\left(t_{M C}-t_{V}\right) M C_{0}+t_{V} M C_{0}+t_{I A} I A_{0}-t_{C F} C F_{0}= \\
& \Delta R O=\left(t_{M C}-t_{V}\right) M C_{0}+t_{V}\left(R O_{0}-I A_{0}+C F_{0}\right)+t_{I A} I A_{0}-t_{C F} C F_{0}= \\
& \Delta R O=t_{V} R O_{0}+\left(t_{M C}-t_{V}\right) M C_{0}+\left(t_{I A}-t_{V}\right) I A_{0}+\left(t_{V}-t_{C F}\right) C F_{0}
\end{aligned}
$$

Dado que:

$$
M C_{1}-M C_{0}=V_{1} \overline{m c_{1}}-V_{0} \overline{m c}_{0}
$$

Si se expresan las magnitudes del año 1 como la suma de las del año 0 más la variación experimentada, y se desarrolla el producto de estas sumas:

$$
\begin{aligned}
& \Delta M C=M C_{1}-M C_{0}=V_{1} \overline{m c}_{1}-V_{0} \overline{m c}_{0}=\left[V_{0}+\left(V_{1}-V_{0}\right)\right]\left[\overline{m c}_{0}+\left(\overline{m c}_{1}-\overline{m c}_{0}\right)\right]-V_{0} \overline{m c}_{0}= \\
& \Delta M C=V_{0} \overline{m c}_{0}+\left(V_{1}-V_{0}\right) \overline{m c}{ }_{0}+V_{0}\left(\overline{m c}_{1}-\overline{m c}_{0}\right)+\left(V_{1}-V_{0}\right)\left(\overline{m c}_{1}-\overline{m c}_{0}\right)-V_{0} \overline{m c}_{0}= \\
& \Delta M C=t_{V} M C_{0}+t_{\bar{m}} M C_{0}+t_{V} t_{\overline{m c}} M C_{0} \\
& M C_{1}-M C_{0}-t_{V} M C_{0}=t_{\overline{m c}} M C_{0}+t_{V} t_{\overline{m c}} M C_{0} ; \\
& M C_{1}-M C_{0}=t_{M C} M C_{0} \rightarrow\left(t_{M C}-t_{V}\right) M C_{0}=t_{\overline{m c}} M C_{0}+t_{V} t_{\overline{m c}} M C_{0}
\end{aligned}
$$

Sustituyendo en [II.4]:

$$
\begin{aligned}
& \Delta R O=t_{V} R O_{0}+\left(t_{M C}-t_{V}\right) M C_{0}+\left(t_{I A}-t_{V}\right) I A_{0}+\left(t_{V}-t_{C F}\right) C F_{0}= \\
& \Delta R O=t_{V} R O_{0}+t_{\overline{m c}} M C_{0}+t_{V} t_{\overline{m c}} M C_{0}+\left(t_{I A}-t_{V}\right) I A_{0}+\left(t_{V}-t_{C F}\right) C F_{0}
\end{aligned}
$$

Como $\overline{m c}=1-\overline{c v}=r \cdot \overline{c v}$, y operando de manera similar a la expresada en [II.5]:

$$
\begin{aligned}
& \Delta \overline{m c}=\overline{m c}_{1}-\overline{m c}_{0}=r_{1} \overline{c v}_{1}-r_{0} \overline{c v}_{0}=\left[r_{0}+\left(r_{1}-r_{0}\right)\right]\left[\overline{c v}_{0}+\left(\overline{c v}_{1}-\overline{c v}_{0}\right)\right]-r_{0} \overline{c v}_{0}= \\
& \Delta \overline{m c}=t_{\overline{m c}} \overline{m c}_{0}=r_{0} \overline{c v_{0}}+r_{0}\left(\overline{c v}_{1}-\overline{c v}_{0}\right)+\left(r_{1}-r_{0}\right) \overline{c v_{0}}+\left(r_{1}-r_{0}\right)\left(\overline{c v}_{1}-\overline{c v_{0}}\right)-r_{0} \overline{c v_{0}}=
\end{aligned}
$$


Donde, multiplicando y dividiendo cada sumando por el valor inicial del componente que varía de manera similar a la operada en [II.3], se obtiene:

$$
\begin{aligned}
& \Delta \overline{m c}=t_{\overline{m c}} \overline{m c}_{0}=r_{0}\left(\overline{c v}_{1}-\overline{c v}_{0}\right)+\left(r_{1}-r_{0}\right) \overline{c v}_{0}+\left(r_{1}-r_{0}\right)\left(\overline{c v}_{1}-\overline{c v}_{0}\right)= \\
& \Delta \overline{m c}=t_{\overline{m c}} \overline{m c}_{0}=\frac{\left(r_{1}-r_{0}\right)}{r_{0}} r_{0} \overline{c v}_{0}+\frac{\left(\overline{c v}_{1}-\overline{c v}_{0}\right)}{\overline{c v}_{0}} r_{0} \overline{c v}_{0}+\frac{\left(r_{1}-r_{0}\right)}{r_{0}} \frac{\left(\overline{c v}_{1}-\overline{c v}_{0}\right)}{\overline{c v}_{0}} r_{0} \overline{c v_{0}}= \\
& t_{\overline{m c}} \overline{m c}_{0}=t_{r} \overline{m c}_{0}+t_{\overline{c v}} \overline{m c}_{0}+t_{r} t_{\overline{c v}} \overline{m c}_{0}
\end{aligned}
$$

Multiplicando por $V_{0}$, y sustituyendo en [II.5] y [II.6]:

$$
\begin{aligned}
& V_{0} t_{\overline{m c}} \overline{m c}_{0}=t_{\overline{m c}} M C_{0}=t_{r} M C_{0}+t_{\overline{c v}} M C_{0}+t_{r} t_{\overline{c v}} M C_{0} \\
& \left(t_{M C}-t_{V}\right) M C_{0}=t_{\overline{m c}} M C_{0}+t_{V} t_{\overline{m c}} M C_{0}=\left(t_{r}+t_{\overline{c v}}+t_{r} t_{\overline{c v}}\right) M C_{0}+t_{V}\left(t_{r}+t_{\overline{c v}}+t_{r} t_{\overline{c v}}\right) M C_{0} \\
& \Delta R O=t_{V} R O_{0}+\left(t_{r}+t_{\overline{c v}}+t_{r} t_{\overline{c v}}\right) M C_{0}+t_{V}\left(t_{r}+t_{c v}+t_{r} t_{\overline{c v}}\right) M C_{0}+\left(t_{I A}-t_{V}\right) I A_{0}+\left(t_{V}-t_{C F}\right) C F_{0}
\end{aligned}
$$

Asimismo, a partir de [II.5] se obtiene la relación entre las tasas de variación de los márgenes medio y total:

$$
\begin{aligned}
& t_{M C} M C_{0}-t_{V} M C_{0}=t_{\overline{m c}} M C_{0}+t_{V} t_{\overline{m c}} M C_{0} \rightarrow t_{M C}-t_{V}=t_{\overline{m c}}\left(1+t_{V}\right) \\
& t_{\overline{m c}}=\frac{t_{M C}-t_{V}}{1+t_{V}}
\end{aligned}
$$

Igualmente, operando de forma similar a la efectuada en [II.5] y [II.7]:

$$
\begin{aligned}
& C V_{1}-C V_{0}=t_{C V} C V_{0}=V_{1} \overline{c v}_{1}-V_{0} \overline{c v}_{0}=\left(V_{1}-V_{0}\right) \overline{c v_{0}}+\left(\overline{c v}_{1}-\overline{c v}_{0}\right) V_{0}+\left(V_{1}-V_{0}\right)\left(\overline{c v}_{1}-\overline{c v}_{0}\right)= \\
& t_{C V} C V_{0}=t_{V} C V_{0}+t_{c v} C V_{0}+t_{V} t_{c v} C V_{0} \rightarrow t_{C V}-t_{V}=t_{\overline{c v}}\left(1+t_{V}\right) \\
& t_{\overline{c v}}=\frac{t_{C V}-t_{V}}{1+t_{V}}
\end{aligned}
$$

